\title{
Estudo do Efeito do Tipo de Polipropileno na Fotodegradação da Blenda Polipropileno/Poliestireno de Alto Impacto
}

\author{
Laércio L. Fernandes, Cássia A. de Freitas, Nicole R. Demarquette \\ Departamento de Engenharia Metalúrgica e Materiais, USP \\ Guilhermino J. M. Fechine \\ Departamento de Engenharia de Materiais, Mackenzie
}

\begin{abstract}
Resumo: Este trabalho visa avaliar a influência do tipo de polipropileno no comportamento da blenda polipropileno/poliestireno de alto impacto (PP/HIPS) quando exposta à radiação UV. Foram usados uma resina virgem de PP (PPv) e outra reprocessada (PPrep). Inicialmente, avaliou-se o comportamento individual dos componentes da blenda, HIPS, PPv e PPrep, quando submetidos à radiação UV por até 15 semanas de exposição. As técnicas de caracterização utilizadas para monitorar o desempenho tanto das resinas individualmente quanto das blendas submetidas à radiação UV foram: propriedades mecânicas (tração e impacto), medidas de índice de fluidez (MFI), análise térmica (DSC), espectroscopia no infravermelho (FTIR) e microscopia eletrônica de varredura (MEV). A partir dos resultados com essas técnicas verificou-se que o PPv foi a resina mais afetada pela radiação e consequentemente as blendas preparadas com esse PP também foram mais sensíveis à fotodegradação do que as demais. Em termos de fotoestabilização este resultado mostra-se interessante, já que as blendas PP/HIPS preparadas com uma resina previamente degradada necessitariam de menores teores de aditivos do que esse mesmo tipo de blenda preparada com resina virgem.
\end{abstract}

Palavras-chave: Polipropileno, poliestireno de alto impacto, blenda, fotodegradação.

\section{Influence of the Type of Polypropylene on the Photodegradation of Blends of Polypropylene/High Impact Polystyrene}

Abstract: The influence from the type of polypropylene on the photodegradation resistance of blends of polypropylene/high impact polystyrene (PP/HIPS) was studied. A virgin polypropylene (PPv) and another sample that suffered prior thermo-mechanical degradation (PPrep) were used. All materials, components of the blends and blends were exposed to UV radiation for up to 15 weeks. They were characterized as a function of time of UV exposure using differential scanning calorimetry (DSC), infrared spectroscopy (FTIR) and scanning electron microscopy (SEM). The mechanical (tensile and impact) properties and melt flow indexes of the materials were also evaluated. The experimental results revealed that PPv resin and its blends were more sensitive to UV radiation than PPrep and its blends. In terms of photostabilization this finding is very interesting, since PP/HIPS blends prepared with a previously degraded polyolefin would require smaller amounts of additives than the same kind of blends prepared with virgin resin.

Keywords: Polypropylene, high impact polystyrene, blend, photodegradation.

\section{Introdução}

O interesse no estudo das blendas poliméricas tem aumentado a cada ano. Um dos grandes motivos para esse crescimento se dá pela vantagem: 1) tecnológica: as blendas possuem a capacidade de combinar as propriedades de diferentes componentes de modo a obter excelentes propriedades mecânicas, térmicas e químicas, e 2) financeira: o custo de desenvolvimento de uma blenda é menor que o custo de desenvolvimento de um novo polímero. Porém o desenvolvimento de um novo material não se restringe apenas a sua obtenção, é preciso também compreender suas características físicas e químicas com o intuito de definir sua aplicação. Assim, para delinear melhor a aplicabilidade do material é necessário entender como a blenda se comportará diante situações reais de uso. Uma das maneiras para avaliar o desempenho desses materiais em situações reais de uso é expô-los à radiação UV.

Sob a ação da radiação solar os polímeros sofrem uma série de reações químicas que os levam a degradação. Em sua maioria a degradação acarreta diminuição nas propriedades mecânicas, alteração de cor e formação de fissuras devido à cisão de cadeia sofrida pelo polímero ${ }^{[1]}$. Partindo dessas afirmações, fica claro que o conhecimento da deterioração causada pelo meio ambiente nas propriedades do material é um aspecto fundamental para que se possa projetar componentes adequados para as aplicações específicas. A necessidade de se prever a vida útil de produtos quando expostos à radiação UV tem incentivado muitos estudos de envelhecimento acelerado em laboratório ${ }^{[2-6]}$.

Apesar dos mecanismos de fotodegradação da maioria dos polímeros serem bem estabelecidos, o comportamento das blendas poliméricas frente à radiação UV ainda é um campo a ser explorado. Alguns tipos de polímeros, como o polietileno, polipropileno, poliestireno e poli(tereftalato de etileno), foram extensivamente estudados ao longo dos anos, e os mecanismos de fotodegradação e fotoestabilização, bem como os fatores que controlam a cinética do processo e mudanças nas propriedades do produto estão razoavelmente bem estabelecidos ${ }^{[7-14]}$. Enquanto isso, no caso de blendas poliméricas, poucos estudos na área de fotodegradação podem ser encontrados na literatura ${ }^{[15-18]}$. Polipropileno (PP) e o poliestireno de alto impacto (HIPS) são usados em larga variedade de produtos. Alguns trabalhos têm sido direcionados ao estudo da preparação de blendas a partir de resinas virgens destes dois polímeros ${ }^{[19,20]}$ com a intenção de melhorar o desempenho do

Autor para correspondência: Guilhermino J. M. Fechine, Departamento de Engenharia de Materiais, Universidade Presbiteriana Mackenzie,

Rua da Consolação, 930, Consolação, CEP: 01302-907, São Paulo, SP, Brasil, e-mail: guilherminojmf@mackenzie.br 
PP com relação a resistência ao impacto. Entretanto, a utilização de materiais reciclados na preparação de blendas também é fonte de diversos estudos, tanto de cunho social como científico. Fechine et al. $^{[21]}$ recentemente publicaram um artigo sobre o comportamento da blenda PP/HIPS frente à radiação UV, avaliando propriedades mecânicas, mecanismo de fratura, índice de fluidez e presença de agente compatibilizante. Neste estudo foi utilizado um PP reprocessado. Observou-se que o aumento da concentração de HIPS e presença do agente compatibilizante (SBS) tornou a blenda menos sensível à radiação UV. A partir dos resultados obtidos por Fechine et al. um questionamento vem a tona: Caso o PP usado fosse um material virgem, como seria o comportamento desta blenda frente à radiação UV? Sendo assim, este trabalho teve como objetivo avaliar a diferença do comportamento da blenda PP/HIPS frente à radiação UV quando se usa dois tipos de polipropileno, um virgem (PPv) e outro reprocessado (PPrep). Esse estudo será embasado através do monitoramento de propriedades térmicas, propriedades mecânicas (tração e impacto), modificações químicas (Espectroscopia no infravermelho-FTIR), Medidas de Índice de Fluidez (MFI) e análise de superfície de fratura de corpos-de-prova de impacto (Microscopia Eletrônica de Varredura - MEV).

\section{Experimental}

\section{Materiais e métodos}

Neste trabalho utilizou-se um polipropileno virgem com índice de fluidez $1,3 \mathrm{~g} / 10 \mathrm{~min}$, densidade igual a $0,90 \mathrm{~g} \cdot \mathrm{cm}^{-1}$ a $25^{\circ} \mathrm{C}$, que daqui por diante será denominado como PPv. O PP reprocessado (PPrep) foi obtido através de cinco extrusões seguidas em uma extrusora dupla rosca Haake, modelo Rheomix PTW 16, acoplada a um reômetro de Torque, modelo Haake Polylab 900, com as seguintes condições de processamento: temperaturas das zonas foram de 190-200-220-220-220 ${ }^{\circ} \mathrm{C}$ e rotação da rosca igual a $100 \mathrm{rpm}$. O poliestireno de alto impacto usado possuía índice de fluidez igual a 11,0 g/10 min. A preparação das blendas poliméricas foi feita em uma extrusora de dupla rosca Haake, modelo Rheomix PTW 16, acoplada a um reômetro de Torque, modelo Haake Polylab 900. O perfil de temperaturas utilizadas ao longo da rosca foi 180-200200-200 ${ }^{\circ} \mathrm{C}$, com rotação de $70 \mathrm{rpm}$. Foram preparadas blendas PP/ HIPS, com PP virgem e reprocessado, com concentrações de HIPS variando de 10 a $30 \%$ em peso. A confecção dos corpos de prova de tração e impacto foi feita através de moldagens por injeção em uma máquina da Demag Ergotech, seguindo as normas da ASTM D638 e D256, respectivamente.

A exposição artificial das amostras foi feita numa câmara de envelhecimento da marca Q-Lab que utiliza lâmpadas fluorescentes UVA, com controle de radiação, temperatura e ambiente de condensação. O ciclo definido da câmara foi de: 8 horas sob luz UV em $60{ }^{\circ} \mathrm{C}$ e 4 horas sem luz em condensação a $50{ }^{\circ} \mathrm{C}$. A intensidade de radiação que alcança a superfície das amostras é de $0,89 \mathrm{Wm}^{-2}$. Sendo assim a exposição seguiu a norma ASTM G-154. As amostras foram expostas por tempos de até 15 semanas.

Todas as amostras foram caracterizadas em função do tempo de exposição por espectroscopia de infravermelho (FTIR), análise térmica (DSC) e superfície de fratura dos corpos-de-prova de impacto via microscopia eletrônica de varredura (MEV). As propriedades mecânicas assim como o MFI dos materiais expostos também foram avaliados. As condições de ensaios são apresentadas a seguir.

As análises de FTIR foram feitas em um Espectrofotômetro NICOLET, Magna 560. As amostras foram obtidas em forma de discos em uma prensa hidráulica da marca Carver, modelo 3393. Os discos continham uma quantidade de $2 \mathrm{mg}$ de $\mathrm{KBr}$ e $1 \%$ em massa de polímero. As análises térmicas foram realizadas num DSC50 da Shimadzu sob taxa de aquecimento igual a $10{ }^{\circ} \mathrm{C} / \mathrm{min}$. Os resultados que serão apresentados são referentes as curvas obtidas na primeira corrida de aquecimento, já que são nessas curvas que estão contidas ás mudanças ocorridas no polímero durante o processo fotodegradativo. As análises de Microscopia Eletrônica de Varredura (MEV) foram efetuadas num microscópio Philips, modelo XL 30, com voltagem de aceleração de $15-20 \mathrm{kV}$ e imagem formada por elétrons secundários. A preparação das amostras foi feita através de sputtering com ouro na superfície de fratura dos corpos de prova após testes de impacto. Os ensaios de tração foram realizados em duas fases em um equipamento Kratos K10.000MP com célula de carga de $500 \mathrm{kgf}$. Na primeira fase a velocidade utilizada foi de $\mathrm{mm} / \mathrm{min}$ até o deslocamento $0,4 \%$ de deformação para o cálculo de Módulo de Elasticidade. Após o término dessa fase, retornou a deformação de $0 \%$. Na segunda fase a velocidade de ensaio foi de $20 \mathrm{~mm} / \mathrm{min}$ até a ruptura do corpo de prova. Os ensaios de impacto foram realizados em uma máquina Tinus Olsen Model Impact 104, à temperatura ambiente. Para cada amostra foram realizados 6 ensaios com corpos de prova sem entalhe. As Medidas de Índice de Fluidez foram realizadas utilizando um plastômetro Ceast Melt Flow - Modular Line. Foi usado um peso de $5 \mathrm{~kg}$ e temperatura de $200{ }^{\circ} \mathrm{C}$. As condições experimentais usadas foram as mesmas para os polímeros estudados individualmente e blendas, para que fosse possível comparar os resultados obtidos nesse teste.

\section{Resultados e Discussão}

Antes de analisar-se o efeito do tipo de polipropileno (virgem e reprocessado) no comportamento da blenda PP/HIPS frente à radiação UV , é necessário avaliar o comportamento individual dos componentes formadores desta blenda. Os resultados referentes ao PPrep e o HIPS já foram apresentados por Fechine et al. em outra publicação ${ }^{[21]}$. Neste trabalho apenas um resumo dos resultados será apresentado. Contudo, no estudo apresentado aqui se fazem necessárias uma comparação do desempenho dos dois tipos de polipropileno assim como do HIPS frente ao UV.

A Figura 1 mostra o efeito do tempo de exposição à radiação UV no módulo de elasticidade, tensão na ruptura, alongamento máximo e resistência ao impacto do HIPS, PPrep e PPv. Observa-se que os resultados obtidos para o PPrep e o PPv são característicos de um polímero que sofreu degradação em decorrência de cisão de cadeias com a diminuição dessas propriedades ${ }^{[10,22]}$. Um fato interessante, que irá se repetir em todas as propriedades mecânicas avaliadas pelo ensaio de tração é que foi impossível realizar o teste com o PPv com 15 semanas de exposição, pois a amostra se encontrava num nível de degradação que tornou impossível posicioná-la na garra sem que a mesma se rompesse. Isso ocorreu porque o nível de degradação do PP virgem chegou a um estágio tão alto que não foi possível manuseá-lo. No caso do HIPS, observa-se um pequeno aumento no módulo de elasticidade, provavelmente, devido a reações de reticulações que podem ocorrer durante o processo fotodegradativo da fase borrachosa presente neste polímero ${ }^{[23-26]}$. Aqui vale ressaltar que o módulo de elasticidade é obtido numa faixa de deformação muito pequena (região elástica), e nessa faixa tanto reações de cisão como de reticulação, mesmo que em pequeno grau de intensidade, são refletidas nessa propriedade. No caso da tensão e alongamento na ruptura observa-se que os valores diminuem significativamente com o aumento do tempo de exposição para todas as amostras. Diferente do módulo de elasticidade, os reflexos das reações de cisão de cadeia e/ou reticulação nas propriedades de tensão e alongamento na ruptura, TR e $\varepsilon_{\text {máx }}$ só são significativos em níveis mais altos. A partir dos resultados apresentados nestas figuras observa-se um 


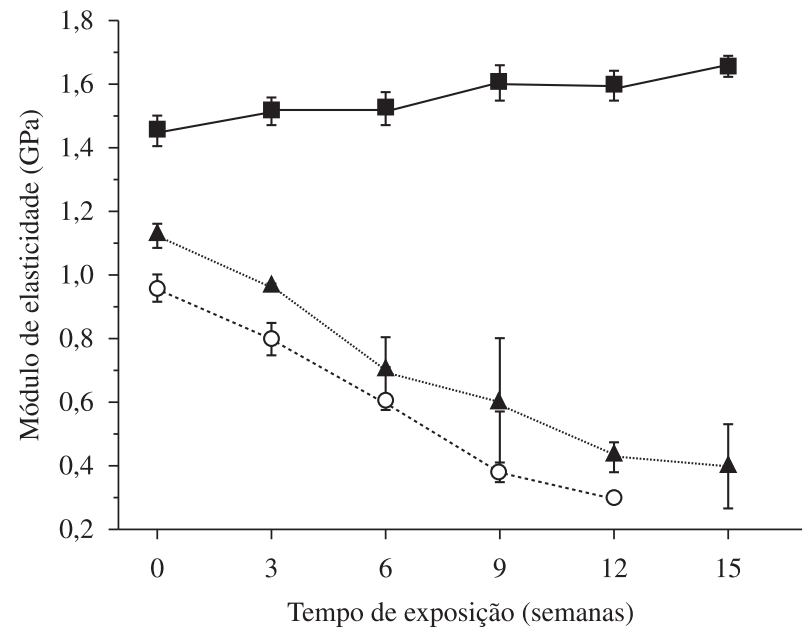

(a)

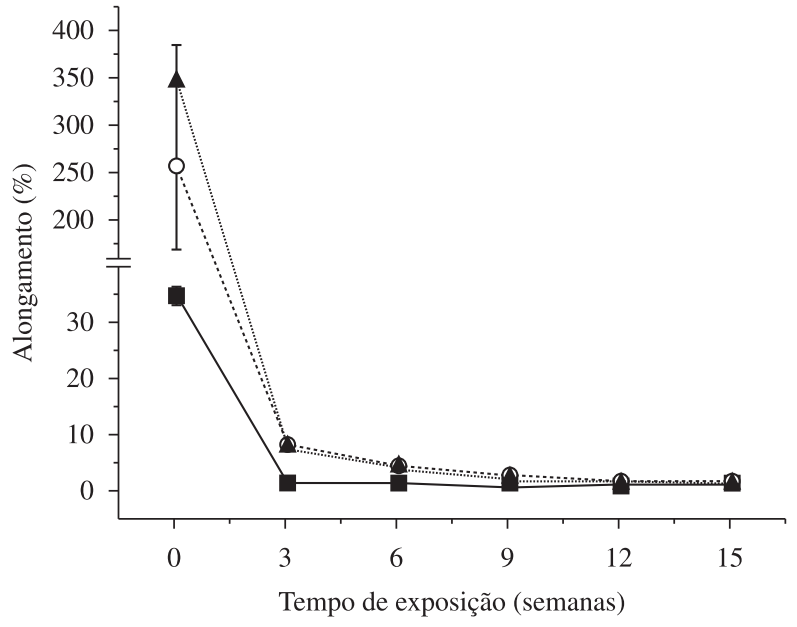

(c)

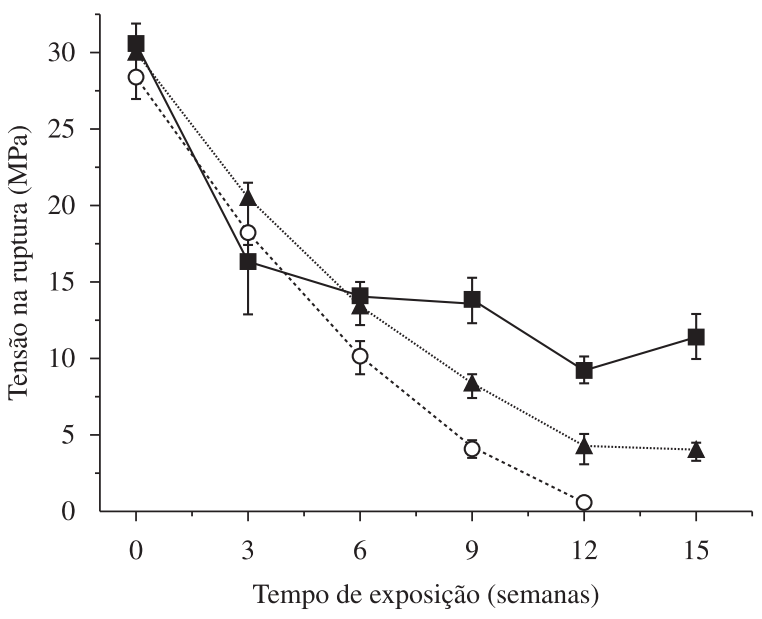

(b)

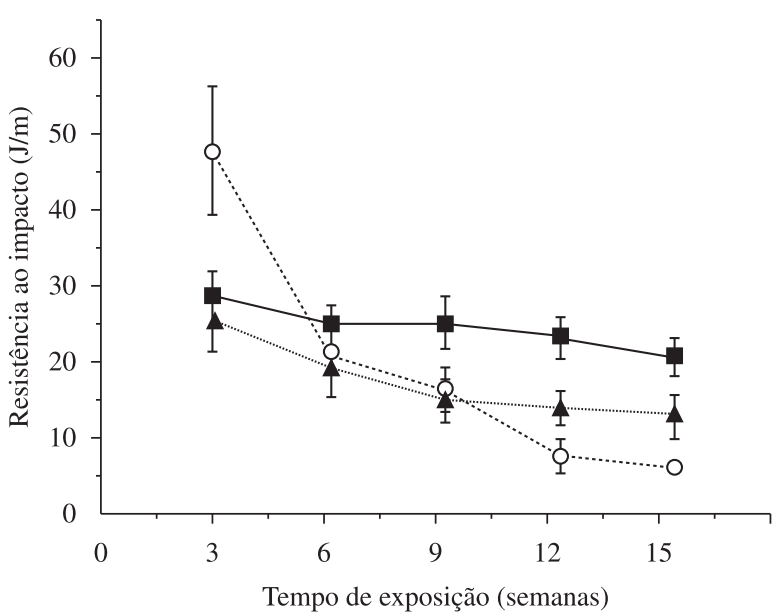

(d)

HIPS $\quad \cdots$ - - PPv $\quad \cdots \cdots \cdots$ PPrep

Figura 1. Efeito do tempo de exposição nos valores médios do: a) Módulo de Elasticidade, b) tensão na ruptura, c) alongamento máximo, e d) resistência ao impacto para o HIPS, PPv e PPrep. Os dados para o PPrep e HIPS já foram apresentados ${ }^{[21]}$ e são reportados aqui somente para fim de comparação.

indicativo de que os três materiais sofreram reações oxidativas que acarretaram em cisão de cadeias. Vale ressaltar que as reações foto-oxidativas ocorrem em regiões com maior disponibilidade de oxigênio, ou seja, na região amorfa. Os resultados indicam que os PP's são significativamente mais sensíveis as cisões de cadeias, tanto para TR como para $\varepsilon_{\text {máx }}$. Esse fato pode ser explicado pelo fato de que a parte amorfa do polipropileno se localizar na região das moléculas atadoras (região interesferulítica).

A Figura 1d apresenta os resultados de resistência ao impacto dos diversos materiais antes de serem incorporados em blendas (note que os corpos de prova não foram entalhados para os testes de impacto). Os materiais não-expostos não romperam após ensaio, portanto não está reportado nenhum dado para o tempo zero de exposição. Para os materiais não-expostos foi usado um pendulo de $22 \mathrm{~J}$, isso significa que os corpos-de-prova não romperam mesmo sendo submetidos a $6.000 \mathrm{~J} \cdot \mathrm{m}^{-1}$. Observa-se na figura a mesma tendência das TR e $\varepsilon_{\text {máx }}$, ou seja, o valor da resistência ao impacto para três amostras diminuiu com o aumento do tempo de exposição. Este comportamento é típico de materiais que sofrem cisão molecular.
Os resultados apresentados na Figura 1 mostram que $o$ PP reprocessado possui um comportamento diferente frente a radiação UV do que o PP virgem: o PP virgem apresenta uma queda nas propriedades mecânicas com o tempo de exposição superior ao PP reprocessado. Por exemplo, a resistência ao impacto do PPv caiou em $88 \%$ após exposição de 15 semanas ao UV enquanto a resistência ao impacto do PP reprocessado caiu de 55\%. Esse fato, possivelmente, ocorreu porque o PP reprocessado, por já se apresentar amarelado, apresenta um comportamento completamente diferente do PP virgem, quando se trata de nível de penetração de radiação UV na amostra. Ou seja, a profundidade de penetração da radiação UV pode ser inferior no PP reprocessado, uma vez que já existem maiores quantidades de grupos cromóforos na superfície do material degradado, que diminui a transmitância da radiação para o interior da amostra, conseqüentemente, menos danos são observados devido à exposição à radiação UV ${ }^{[18]}$. Esse fato corrobora com os dados obtidos para o HIPS, o qual se apresenta como um material opaco, e consequentemente, no qual pouca radiação é transmitida para o interior da amostras, e por isso os danos causados são inferiores as amostras de polipropileno. 
A diminuição dos valores das propriedades TR e $\varepsilon_{\text {máx }}$ com o tempo de exposição pode ser explicada pela queda da massa molar dos polímeros expostos ao UV e pela sua oxidação que pode ser avaliada por analise química. A avaliação do índice de fluidez (MFI) dos polímeros pode fornecer informações sobre a queda de massa molar do PP. A Figura 2 apresenta o MFI para os três polímeros estudados em função do tempo de exposição ao UV. Pode ser visto que para os três polímeros estudados o MFI cresce com o aumento do tempo de exposição indicando que os três polímeros sofrem cisão de cadeias. Este aumento de MFI é muito menor para o HIPS. Para 0 semanas de exposição o PPrep possui um índice de fluidez maior que o do PPv devido a degradação termo-mecânica sofrida durante as diversas extrusões. Esta tendência é revertida conforme o material é exposto a UV indicando que o PPrep é mais resistente a degradação UV do que o PPv.

Pode também ser visto na Figura 2 que após 9 semanas de exposição não foi possível obter medidas de índice de fluidez para o PPv e para o PPrep. A impossibilidade de avaliação do MFI

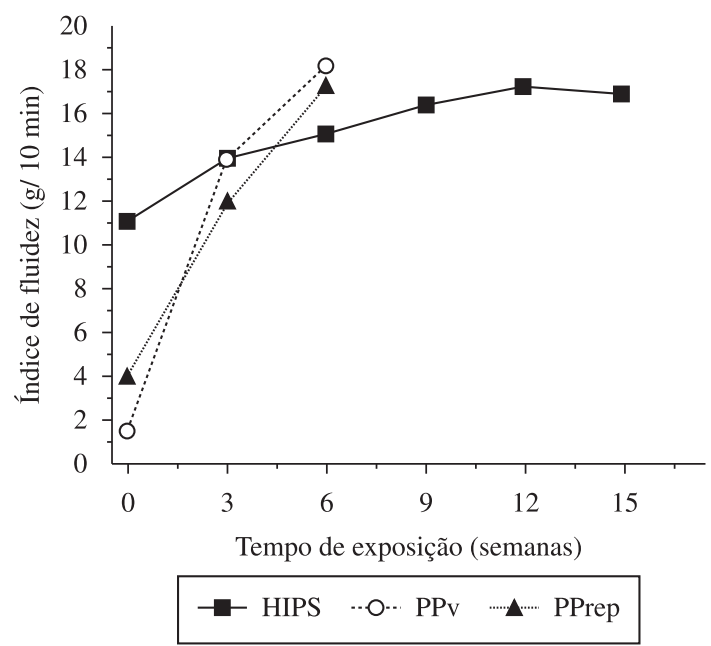

Figura 2. Efeito do tempo de exposição no MFI do HIPS, PPv e PPrep. Os dados para o PPrep e HIPS já foram apresentados ${ }^{[21]}$.

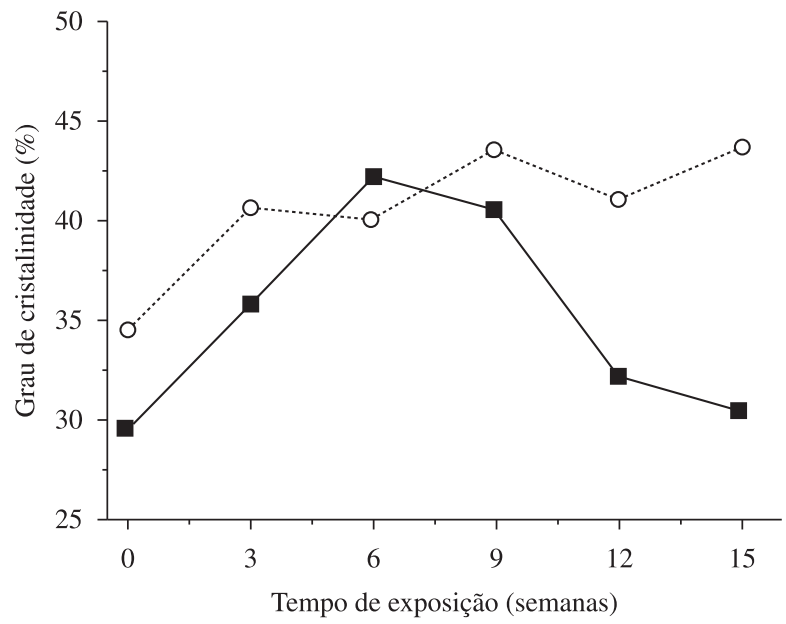

(a) para estas amostras pode ser oriunda da pos-degradação que os materiais sofrem durante o ensaio de MFI. Durante um ensaio de MFI a amostra é submetida a altas temperaturas por um intervalo de tempo relativamente longo (6 a 7 minutos) em atmosfera oxidativa. Durante este tempo, deve ocorrer degradação térmica in situ. Provavelmente, nas amostras já fotodegradadas, a presença de grupos hidroperóxidos e carbonila em altas concentrações, as tornam mais susceptíveis a esse tipo de degradação durante o ensaio de MFI. Uma confirmação para essa suposição são os dados já apresentados para propriedades mecânicas (Figura 1). As amostras para as quais não foi possível obter dados de MFI apresentaram resultados inferiores de propriedades mecânicas.

Uma das maneiras para avaliar as modificações químicas durante o processo degradativo do PP é a análise quantitativa do índice de carbonila (IC). Esse índice é dado pela divisão da intensidade da absorbância do grupo carbonila $\left(1720 \mathrm{~cm}^{-1}\right)$ pela intensidade da absorbância do pico de referência $\left(2750 \mathrm{~cm}^{-1}\right)^{[1]}$. A Figura 3 apresenta os resultados obtidos de IC para o PPrep e PPv

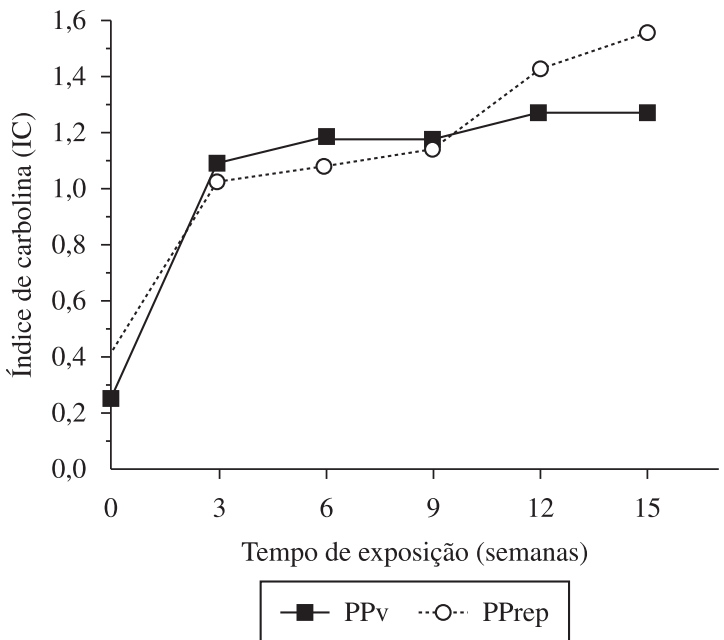

Figura 3. Efeito do tempo de exposição no índice de carbonila para o PPrep e para o PPv.

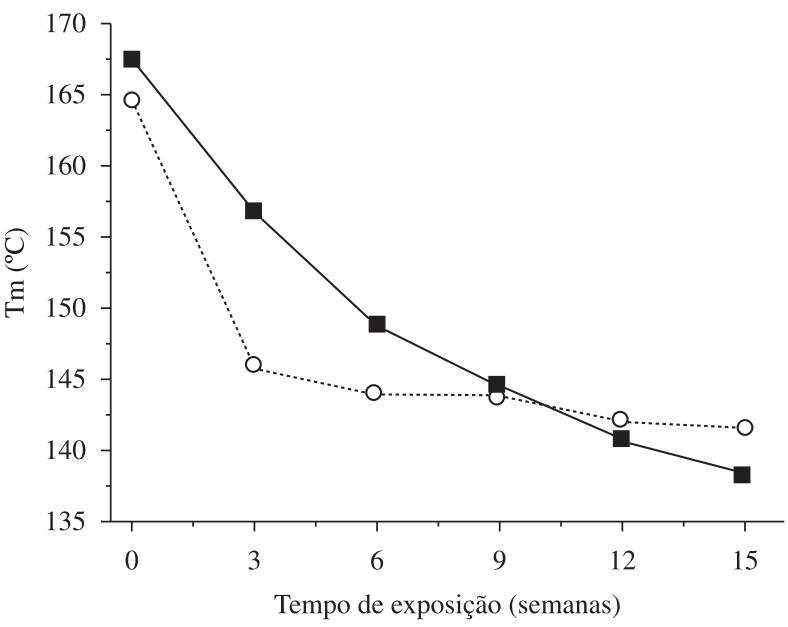

(b)

PPv $\quad \cdots$ - - PPrep

Figura 4. Efeito do tempo de exposição no: a) grau de cristalinidade e b) temperatura de fusão cristalina do PPv e do PPrep. 
em função do tempo de exposição ao UV. Nesta figura pode-se notar que o PP previamente termodegradado possui maior IC do que o PPv quando esse não foi ainda exposto à radiação UV. Isso confirma a degradação prévia sofrida por esse material. Com o aumento do tempo de exposição, nota-se uma mesma tendência no aumento do $\mathrm{IC}$, porém no final de 15 semanas o índice de carbonila do $\mathrm{PPv}$ é superior ao do PPrep. Esses resultados explicam os resultados obtidos com os ensaios mecânicos, ou seja, o aumento no índice de carbonila resulta em queda nas propriedades mecânicas já que a formação do grupo carbonila é o produto resultante das reações de cisão de cadeia do $\mathrm{PP}^{[1]}$.

A Figura 4 mostra os resultados da temperatura de fusão e da cristalinidade do PPrep e do PPv, quando expostos à radiação sob diferentes tempos de exposição. Pode ser visto que para ambas resinas a temperatura de fusão diminui com o tempo de exposição devido às cisões de cadeias ocorridas na superfície lamelar dos cristais $^{[27]}$. Os resultados apresentados na Figura 4 também mostram que a cristalinidade do material aumenta com o tempo de exposição devido ao fenômeno bem conhecido e chamado de quemicristalização. Esta quemicristalização se traduz por um rearranjo molecular das moléculas da região amorfa que sofreram cisão. Contudo, esse rearranjo possui certo limite, já que chegase a um determinado ponto em que o tempo de exposição é tão intenso que a cristalização tende a diminuir, devido ao alto teor de degradação. Este fato ocorre com o PPv após 9 semanas, mas não acontece com o PPrep indicando que o PPrep é mais resistente a degradação UV do que o PPv. Como dito anteriormente, esta melhor resistência ao UV do PPv deve estar relacionada com a diferença de profundidade de penetração da radiação UV nas duas amostras. O PPrep, por estar mais amarelado, é atingido menos pronunciadamente pela radiação UV que o PPv, logo, esse material não atinge o estágio de degradação que leva a uma diminuição da cristalinidade mesmo após 15 semanas de exposição.

A Figura 5 apresenta uma comparação das micrografias dos corpos-de-prova de impacto (região próxima ao local de impacto do pêndulo com a amostra) do PPrep e PPv expostos por 3 e 15 semanas. Vale lembrar que as amostras não-expostas à radiação UV não romperam durante a análise, e, por isso, não são apresentadas as micrografias para essas amostras. Observa-se que após 3 semanas a superfície de fraturas das amostras PPrep e PPv possuem similaridades, apresentando zonas com pouca variação de topografia, caracterizando-se como material frágil. No caso das amostras PPrep e PPv degradadas por 15 semanas, observa-se um alto grau de fragilização das amostras em regiões bem próximas a superfície da amostra, tanto no lado diretamente exposto à radiação quanto do lado contrário. Para esses tempos de exposição, verificouse que a amostra PPv apresenta um grau de deteriorização maior, refletindo em valores menores de resistência ao impacto.

No restante do presente artigo serão apresentados os resultados da blenda PP/HIPS. Estes dados serão apresentados na forma de perda ou ganho (em percentual) das propriedades mecânicas das blendas PP/HIPS, obtidas a partir do PPv e do PPrep. Em

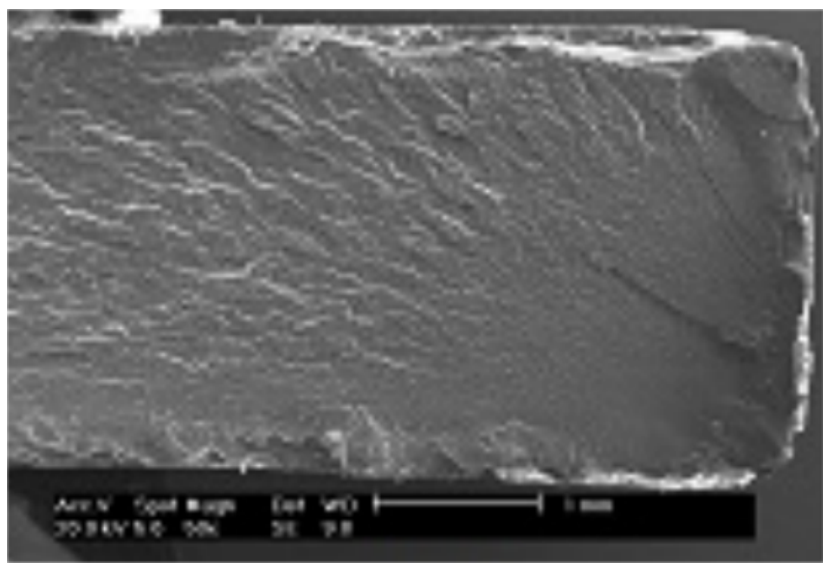

(a)

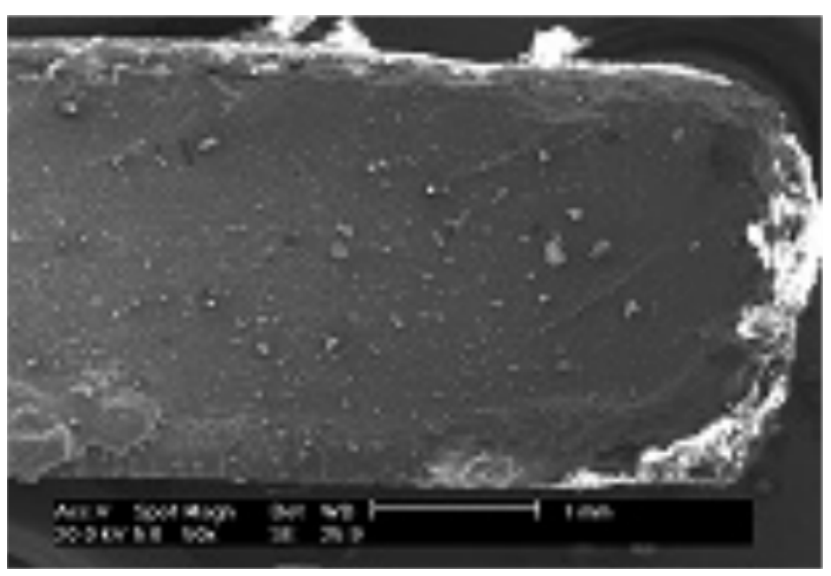

(c)

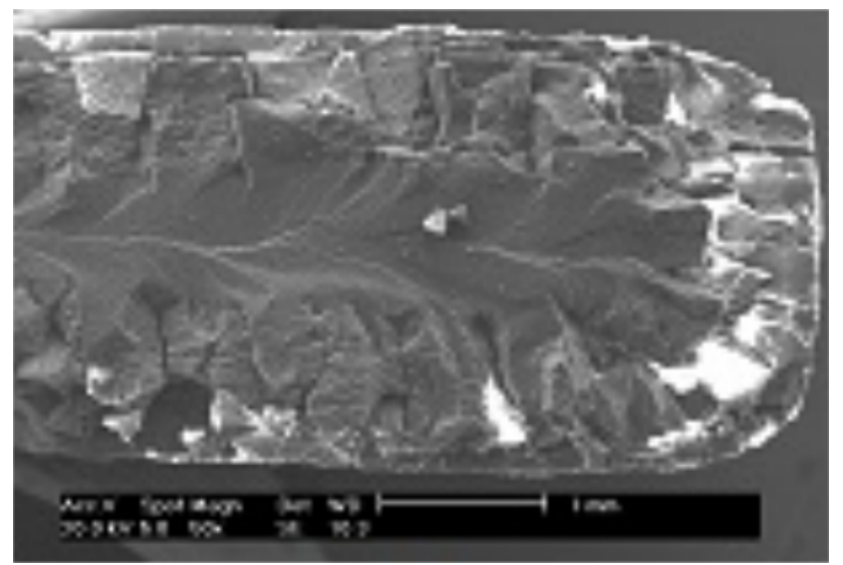

(b)

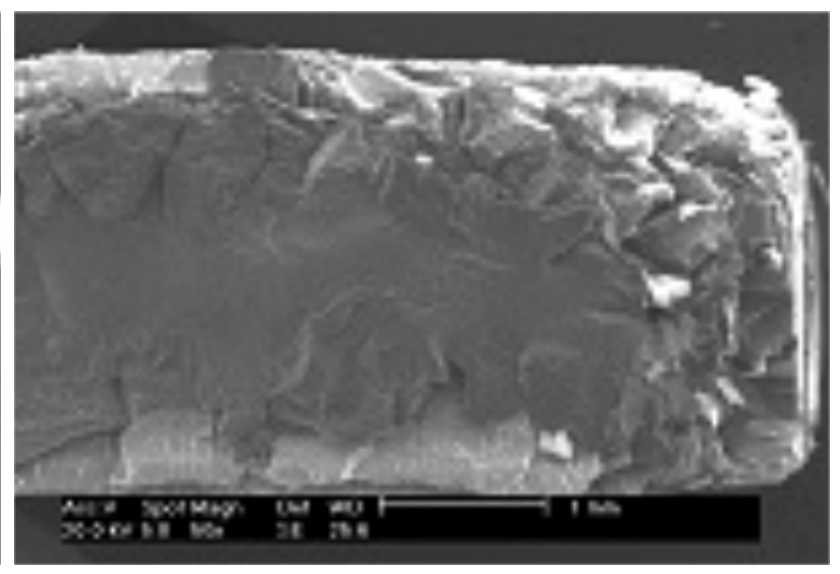

(d)

Figura 5. Micrografias da superfície de fratura de corpos-de-prova de impacto para: a) PPrep exposto por 3 semanas e b) 15 semanas; c) PPv exposto por 3 semanas e d) 15 semanas. 


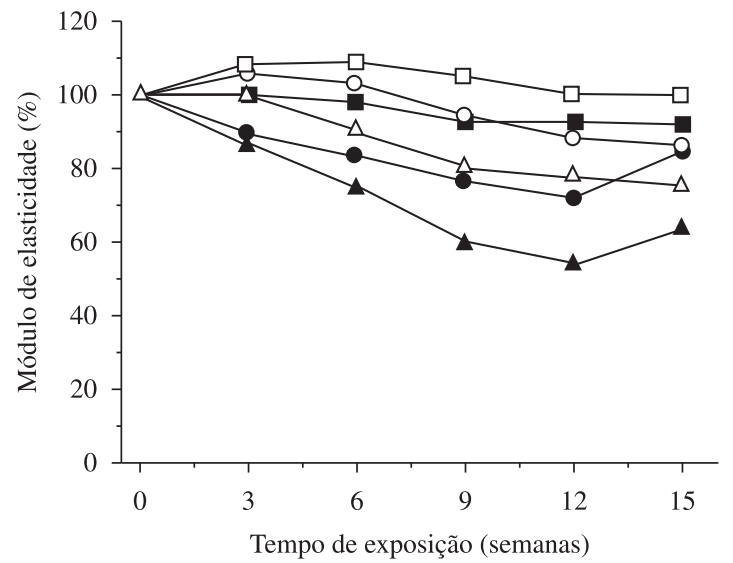

(a)

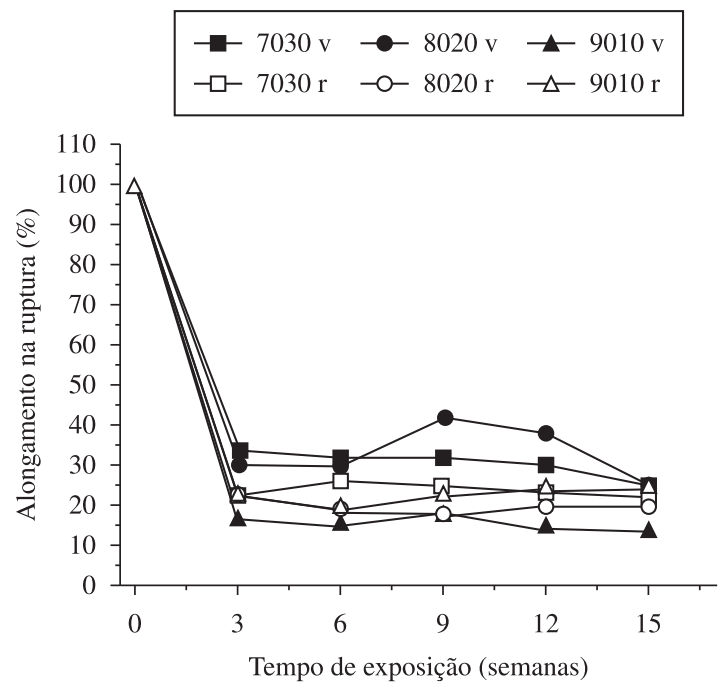

(c)

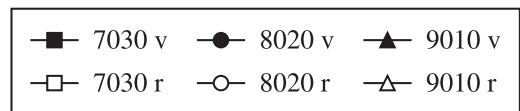

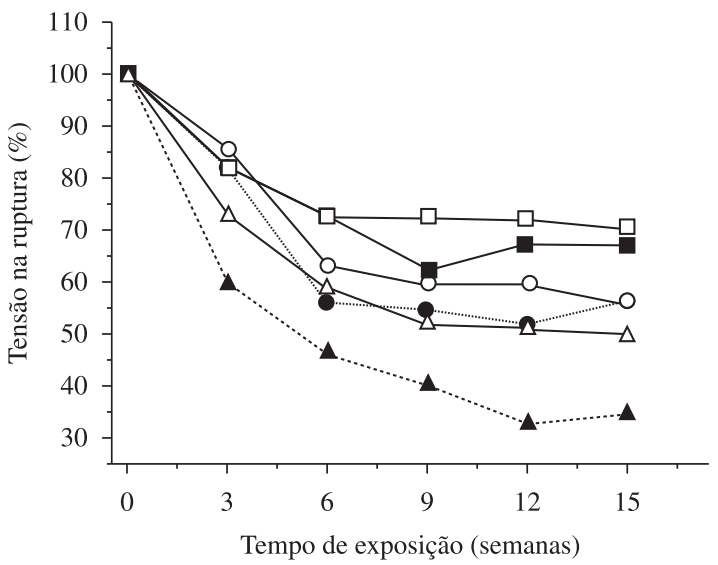

(b)
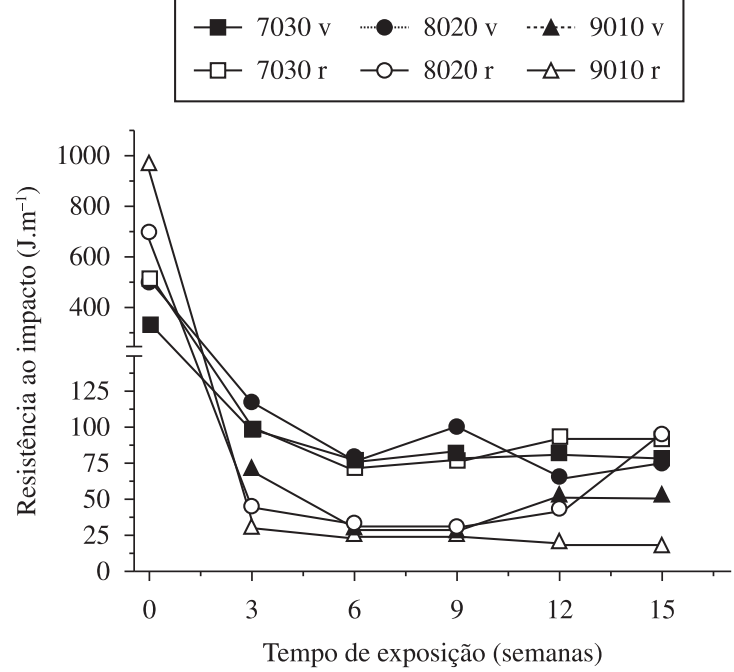

(d)

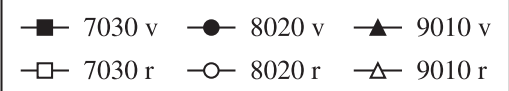

Figura 6. Efeito do tempo de exposição nos valores médios do: a) Módulo de Elasticidade, b) tensão na ruptura, c) alongamento máximo e d) resistência ao impacto para as blendas PP/HIPS. Os dados para as blendas PPrep/HIPS já foram apresentados ${ }^{[21]}$ e são apresentados aqui apenas por fins comparativos.

todas as figuras serão utilizados os índices "v" e "r" nas blendas para identificar as blendas obtidas a partir do PPv e PPrep, respectivamente. A Figura 6 apresenta os resultados de módulo de elasticidade, tensão na ruptura, alongamento máximo e resistência ao impacto obtidos para as blendas PP/HIPS expostas a diferentes tempos de exposição à radiação UV. Em todos os casos a blenda com a presença do PPrep ao final de 15 semanas de exposição sofre menos efeito da radiação UV (relativa ao PPvir), principalmente, a blenda 90/10. Este fato é repetidamente verificado para as outras propriedades, tensão e alongamento na ruptura, como pode ser visto nas Figuras $6 \mathrm{~b}, \mathrm{c}$, respectivamente. No caso dos dados de resistência ao impacto, fica difícil apresentá-lo na forma de perda em percentual, pois a amostra 90/10 não exposta não rompe após o ensaio. Diante desse fato esses resultados estão apresentados em valores nominais de energia $\left(\mathrm{J} . \mathrm{m}^{-1}\right)$, como pode ser visto na Figura 6d. Os resultados indicam que todas as blendas apresentam uma diminuição nos valores de resistência ao impacto com o aumento do tempo de exposição, e essa queda é bem acentuada logo após 3 semanas de exposição. Contudo, quanto maior é o percentual de HIPS nas blendas menor é a queda desta propriedade. Este fato

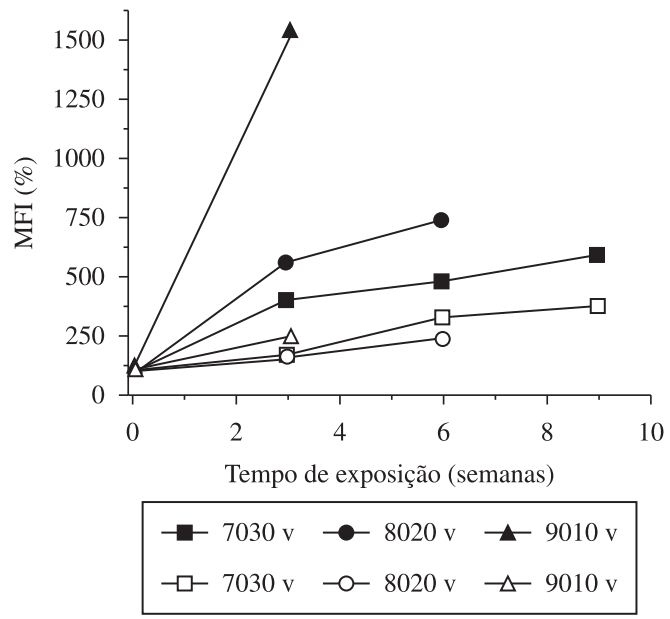

Figura 7. Efeito do tempo de exposição à radiação UV no MFI das blendas PP/ HIPS. Os dados para as blendas PPrep/HIPS já foram apresentados ${ }^{[21]}$ e são apresentados aqui apenas por fins comparativos. 


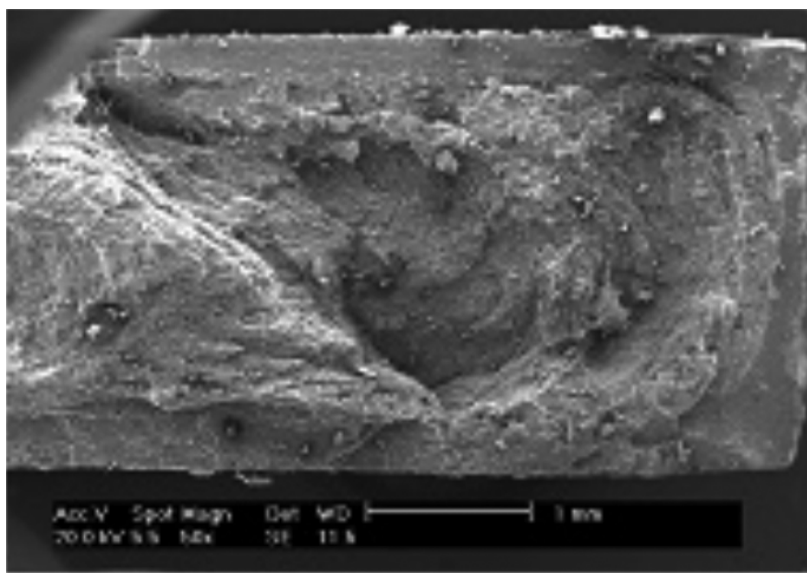

(a)

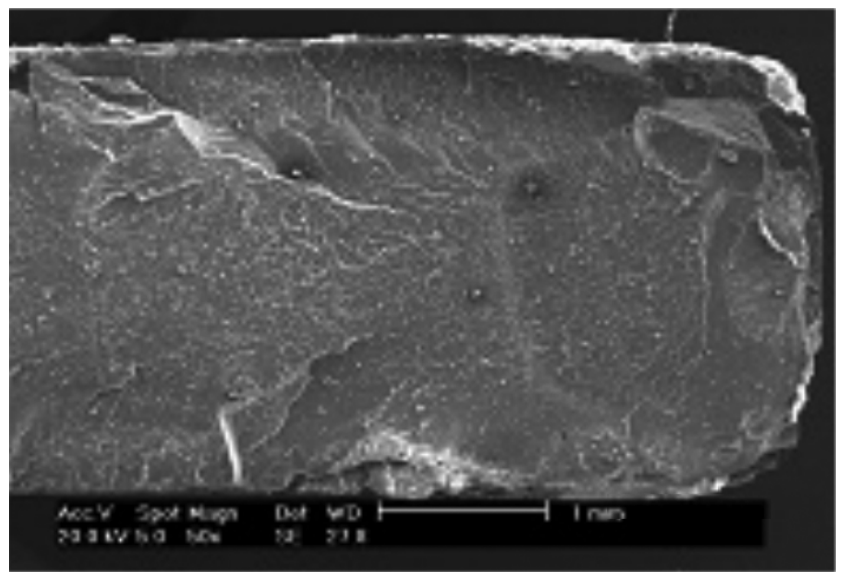

(b)

Figura 8. Micrografias da superfície de fratura de corpos-de-prova de impacto (região próxima a solicitação) expostos por 15 semanas para as blendas a) $70 / 30 \mathrm{v}$ e b) $90 / 1 \mathrm{v}$.

pode ser explicado diante do fato do aumento da fase HIPS também aumenta a opacidade da amostras, sendo assim, os corpos-de-prova ficam menos sensíveis a penetração da radiação UV. No caso do efeito do tipo de PP, a diferença mais significativa é apenas notada para a blenda 90/10. Uma vez que a 90/10 v não rompe antes da exposição à radiação UV, isso significa dizer que a energia para sua ruptura deve ser maior que $6.000 \mathrm{~J}^{\mathrm{m}} \mathrm{m}^{-1}$. Ao final de 15 semanas esse valor cai para em média $55 \mathrm{~J} . \mathrm{m}^{-1}$, enquanto que a blenda 90/10 r ao final de 15 semanas rompe com um valor de $18 \mathrm{~J} . \mathrm{m}^{-1}$, porém, antes da exposição à radiação UV essa amostra precisava de apenas $910 \mathrm{~J} . \mathrm{m}^{-1}$ para se romper. Em termos de percentuais de perda, a blenda 90/10 v sofre uma maior queda. Esse dado corrobora com os demais dados de propriedades mecânicas.

Também foram realizados ensaios de MFI para todas as blendas, como pode ser visto na Figura 7. Os resultados obtidos são reflexos dos dados dos componentes individuais, o mesmo tipo de degradação in situ ocorreu com as blendas. Quanto maior o teor de PP na blenda, maior foi esse fenômeno. Ao se fazer uma comparação entre as blendas com $\mathrm{PPv}$ e PPrep, verifica-se novamente que a blenda preparada com o PP virgem, sofre muito mais danos devido à radiação UV que a preparada com o $\mathrm{PP}$ reprocessado. $\mathrm{O}$ aumento do tempo de exposição gerou valores de MFI para blenda com PPv sempre superiores ao da blenda com PPrep, caracterizando um maior nível de degradação. Este resultado confirma as suposições anteriormente levantadas.

A Figura 8 apresenta as superfícies de fratura das blendas PP/ HIPS, 70/30 e 90/10, expostas à radiação UV por 15 semanas. Verifica-se que o mecanismo de fratura das blendas difere dos componentes individuais. Aqui estão reportadas apenas as micrografias das blendas com o PPv, as com o PPrep são similares e estão reportadas no artigo recém publicado por Fechine et al. ${ }^{[21]}$. Quanto maior a quantidade de HIPS nas blendas mais claramente este fato é verificado. Novamente, a presença do HIPS torna as amostras menos vulneráveis a penetração da radiação UV. E, novamente, verifica-se que a blenda 90/10 é a que mais indica a diferença entre o uso dos diferentes tipos de PP, já que a 90/10 v apresenta-se com uma topografia indicativa de um material com um comportamento mais frágil.

\section{Conclusões}

Este trabalho teve como objetivo avaliar a influência do tipo de polipropileno no comportamento da blenda PP/HIPS frente à radiação UV. A partir dos resultados obtidos neste trabalho algumas conclusões podem ser feitas. Primeiramente, observou-se que há uma grande diferença entre o comportamento dos PP e do HIPS mediante à radiação UV, indicando que a opacidade do HIPS conduziu a um melhor desempenho desse material quando submetido a tempos prolongados de exposição. Entre os polipropilenos, verificou-se que o maior nível de amarelamento do PPrep foi o responsável pela menor penetração da radiação UV na amostra, conduzindo a melhor resistência a radiação UV quando comparado com o PP virgem. Esses resultados refletiram-se no comportamento das blendas PP/ HIPS, para as quais se observou que as blendas produzidas com o PPrep também apresentaram melhores resistência ao UV. Este fato leva a uma conclusão no mínimo interessante. Blendas de PP/HIPS preparadas com uma fase PP previamente degradada necessitariam menores teores de aditivos fotoestabilizantes que blendas preparadas com uma resina de PP virgem. Esse resultado é muito importante para a área de reciclagem. Ou seja, a utilização de PP reciclado na produção de blendas PP/HIPS poderia levar a uma economia no que se diz respeito a aditivação do produto final.

\section{Agradecimentos}

Os autores agradecem a FAPESP, CAPES e ao CNPq.

\section{Referências Bibliográficas}

1. Rabek, J. F. - "Polymer Photodegradation", Chapman \& Hall, London (1995).

2. Fechine, G. J. M. - "Fotodegradação e Fotoestabilização do Poli(tereftalato de etileno)", Tese de Doutorado, Universidade Federal de Pernambuco, Brasil (2001).

3. Rabello, M. S. \& White, J. R. - Plast. Rubber Compos., 25, p.237 (1996).

4. Shyichuk, A. V.; Turton, A. T.; White, J. R. \& Syrotynska, I. D. Polym. Degrad. Stab., 86, p.377 (2004).

5. Gasa, J. V.; Liu, Z \& Shaw, M. T. - Polym. Degrad. Stab., 87, p.77 (2005).

6. Real, L. P.; Gardette, J. L. \& Rocha, A. P. - Polym. Degrad. Stab., 88, p.357 (2005).

7. Fechine, G. J. M. \& Rabello, M. S. - "Sensibilidade de poliolefinas ao envelhecimento natural", in: Anais do $13^{\circ}$ Cbecimat, p.3635, Curitiba - PR (1998).

8. Fechine, G. J. M.; Souto-Maior, R. M. \& Rabello, M. S. - Polym. Degrad. Stab., 75, p.153 (2002). 
9. Fechine, G. J. M.; Catalani, L. H. C. \& Rabello, M. S. - Polymer, 45, p.2303 (2004).

10. Rabello, M. S. \& White, J. R. - Polym. Degrad. Stab., 56, p.55 (1997).

11. Torikai, A.; Shirakawa, H.; Nagaya, S. \& Fueki, K. - J. Appl. Polym. Sci., 40, p.1637 (1990).

12. Tavares, A. C.; Gulmine, J. V.; Lepienski, C. M. \& Akcelrud, L. Polym. Degrad. Stab., 79, p.385 (2003).

13. Pankansen, S.; Kuczynski, J. \& Thomas, J. k. - Macromolecules, 27, p.3773 (1994).

14. Tjandraatmadja, G. F.; Burn, L. S. \& Jollands, M. C. - Polym. Degrad. Stab., 78, p.435 (2002).

15. Torikai, A.; Sekigawa, Y. \& Fueki, K. - Polym. Degrad. Stab., 21, p.43 (1988).

16. Kaczmarek, H.; Kowalonek, J.; Klusek, Z.; Pirzgalski, S. \& Datta, S. J. Polym. Sci. Part B, Polym. Phys., 42, p.585 (2004).

17. Kaczmarek, H.; Podgórski, A. \& Bajer, K. - J. Photochem. Photobiol., A, Chem., 171, p.191 (2004)
18. Waldman, W, R. \& De Paoli, M. A. - Polym. Degrad. Stab., 93, p.273 (2008).

19. Mustafa, S. J.; Azlan, M. R.; Fuad, M. Y. A.; Isshak, Z. A. M. \& Ishiaku, U. S. - J. Appl. Polym. Sci., 82, p.428 (2001).

20. De Melo, T. J. A; Carvalho, L. H.; Calumby, R. B.; Brito, K. G. Q.; D’Almeida, J. R. M. \& Spieth, E. - Polímeros, 10, p.82 (2000).

21. Fernandes, L. L.; Freitas, C. A.; Demarquette, D. R. \& Fechine, G. J. M - J. Appl. Polym. Sci., in press (2010).

22. Fechine, G. J. M. \& Demarquette, N. R. - Polym. Eng. Sci., 48, p.365 (2008).

23. Ghaffar, A.; Scott, A. \& Scott, G. - Eur. Polym. J., 13, p.89 (1977).

24. Ghaffar, A.; Scott, A. \& Scott, G. - Eur. Polym. J., 13, p.83 (1977).

25. Ghaffar, A.; Scott, A. \& Scott, G. - Eur. Polym. J., 12, p.615 (1976).

26. Ghaffar, A.; Scott, A. \& Scott, G. - Eur. Polym. J., 11, p.271 (1975).

27. Rabello, M. S. \& White. J. R. - Polímeros, 7, p.47 (1997).

Enviado: $11 / 08 / 10$

Reenviado: 01/02/11

Aceito: $27 / 04 / 11$ 